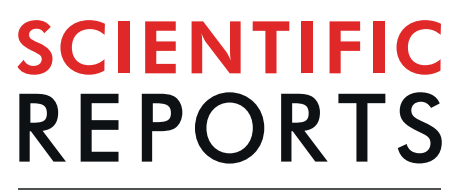

natureresearch

\title{
OPEN Effect of weight change and lifestyle modifications on the development or remission of nonalcoholic fatty liver disease: sex-specific analysis
}

\author{
NaokiYoshioka ${ }^{1,2}$, Masatoshi Ishigami ${ }^{1 *}$, Yasuko Watanabe ${ }^{3}$, Hajime Sumi ${ }^{2}$, Masao Doisaki ${ }^{2}$, \\ Takeo Yamaguchi ${ }^{2}$, Takanori Ito ${ }^{1}$, Yoji Ishizu ${ }^{1}$, Teiji Kuzuya ${ }^{1}$, Takashi Honda ${ }^{1}$, Tetsuya Ishikawa ${ }^{1}{ }^{1}$, \\ Jun-ichi Haruta ${ }^{2} \&$ Mitsuhiro Fujishiro ${ }^{1}$
}

The effects of changes in various lifestyle habits on nonalcoholic fatty liver disease (NAFLD) have not been well elucidated. We aimed to clarify how weight change and lifestyle modifications were associated with the development or remission of NAFLD. In this longitudinal cohort study, we reviewed the periodic health checkup data of 1,421 subjects with no causes of liver disease besides NAFLD who had received at least two health checkups between 2009 and 2018. The prevalence of NAFLD at baseline was $34.1 \%(484 / 1,421)$. During follow-up period $(4.6 \pm 2.8$ years), 104 subjects developed NAFLD and 127 subjects demonstrated NAFLD remission. The frequency of NAFLD development or that of NAFLD remission significantly increased as the larger weight gain or weight loss was, respectively (both, $p<0.001$ ). Approximately $40 \%$ of the subjects who maintained $\geq 1 \% / y e a r$ weight loss achieved NAFLD remission. By multivariate analysis, quitting smoking were independently associated with NAFLD development (adjusted odds ratio [AOR], 2.86; 95\% Cl, 1.24-6.62). Subjects who quit smoking demonstrated large weight gain ( $\geq 1 \% /$ year) significantly more frequently than the other subjects $(p<0.001)$. In sex-specific analysis, starting to exercise was independently associated with NAFLD remission in men (AOR, 2.38; 95\% Cl, 1.25-4.53).

Nonalcoholic fatty liver disease (NAFLD) is one of the most common chronic liver diseases and will become the leading cause of end-stage liver disease in the near future. NAFLD is a global public health concern with a heavy healthcare burden ${ }^{1}$.

NAFLD encompasses a spectrum of progressive pathological conditions, ranging from nonalcoholic fatty liver (NAFL) to steatohepatitis (NASH) ${ }^{2}$. Many clinical trials have shown that weight loss and lifestyle intervention improve markers of liver function and histological features in patients with NAFLD ${ }^{3-13}$. Thus, NAFLD is closely related to lifestyle habits, and is often described as the hepatic manifestation of metabolic syndrome.

NAFLD is reversible to some extent. There have been several studies on lifestyle habits associated with the development or remission of NAFLD ${ }^{14-18}$. However, most of the studies focused only on the lifestyle habits at baseline, but not the changes in lifestyle habits during the study period.

Beginning in April 2008, Japan initiated a change in the national health policy in order to prevent metabolic syndrome. This comprehensive preventive policy involves unprecedented nationwide screening and lifestyle intervention for abdominal obesity, and the effectiveness of the nationwide preventive program has been demonstrated $^{19}$. In addition, various lifestyle habits can be modified as a result of recently growing health consciousness across Japanese society.

${ }^{1}$ Department of Gastroenterology and Hepatology, Nagoya University Graduate School of Medicine, 65 Tsurumacho, Showa-ku, Nagoya, 466-8550, Japan. ${ }^{2}$ Department of Gastroenterology and Hepatology, Japanese Red Cross Nagoya Daiichi Hospital, 3-35 Michishita-cho, Nakamura-ku, Nagoya, 453-8511, Japan. ${ }^{3}$ Health control Center, Japanese Red Cross Nagoya Daiichi Hospital, 3-35 Michishita-cho, Nakamura-ku, Nagoya, 453-8511, Japan. *email: masaishi@med.nagoya-u.ac.jp 


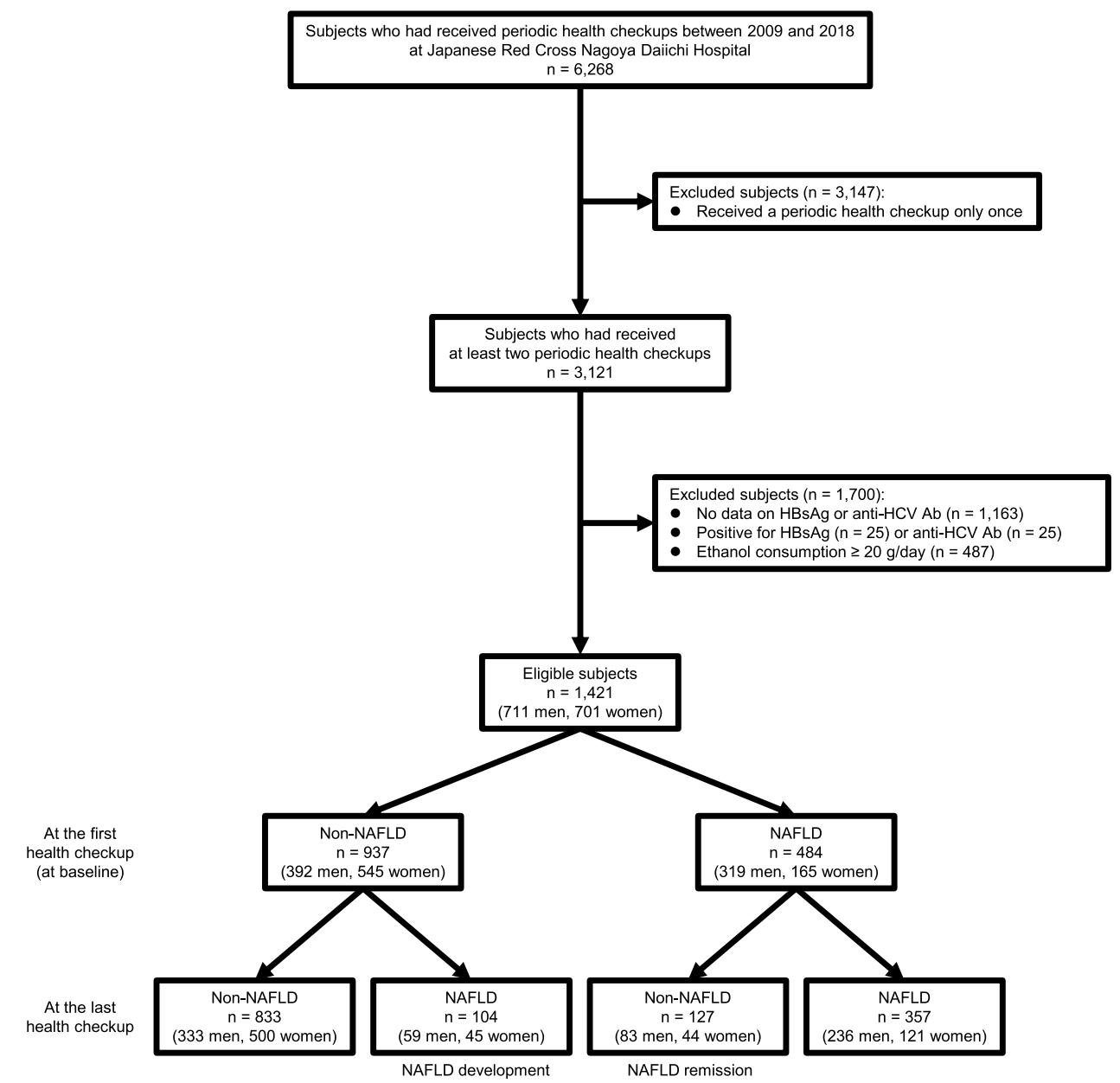

Figure 1. Flow chart of the study population. Eligible subjects comprised 1,421 individuals with no causes of liver disease other than nonalcoholic fatty liver disease (NAFLD) who had received at least two health checkups between 2009 and 2018. Among them, 104 subjects developed NAFLD and 127 subjects demonstrated NAFLD remission during the follow-up period. HBsAg, hepatitis B surface antigen; anti-HCV Ab, anti-hepatitis $\mathrm{C}$ virus antibody.

Understanding the effects of various lifestyle modifications on NAFLD is important for educating the general public as well as for providing guidance to practitioners regarding health checkups. In the present study, we investigated how weight change and lifestyle modifications were associated with the development or remission of NAFLD.

\section{Results}

Study population. In this longitudinal cohort study, we reviewed the health checkup records of 6,268 Japanese adults who had received periodic health checkups between January 2009 and December 2018 at Japanese Red Cross Nagoya Daiichi Hospital (Fig. 1). Among them, 3,121 subjects had received at least two health checkups during this time. Then, subjects who were positive for hepatitis B surface antigen (HBsAg) or anti-hepatitis $\mathrm{C}$ virus antibody (anti-HCV Ab) and those who had not undergone these tests were excluded. Further, subjects who drank $\geq 20 \mathrm{~g} /$ day of ethanol were excluded. Finally, a total of 1,421 subjects ( 711 men and 710 women) was eligible for the study.

Characteristics of subjects at baseline. At the first health checkup (at baseline), 484 of 1,421 (34.1\%) subjects were diagnosed with NAFLD (Fig. 1). In the subjects with NAFLD at baseline, aspartate transaminase (AST), alanine aminotransferase (ALT), and platelet levels, AST to platelet ratio index (APRI), and body mass index (BMI) were significantly higher than those without NAFLD (Table 1). The frequencies of male gender, fatty liver index $(F L I) \geq 30$, hypertension, dyslipidemia, diabetes mellitus, smoking, and eating dinner within 2 hours of going to bed were significantly higher and that of exercise was significantly lower in subjects with NAFLD than those without NAFLD.

Comparison of characteristics of subjects between baseline and the last health checkup. During the follow-up period ( $4.6 \pm 2.8$ years), the number of health checkups which subjects had received was $4.8 \pm 2.7$. During the period, 104 of 937 (11.1\%, 2.4\%/year) subjects without NAFLD at baseline developed NAFLD (Fig. 1). 


\begin{tabular}{|l|l|l|l|l|}
\hline & All $(\mathbf{n}=\mathbf{1 , 4 2 1})$ & $\begin{array}{l}\text { Non-NAFLD } \\
(\mathbf{n}=\mathbf{9 3 7})\end{array}$ & $\begin{array}{l}\text { NAFLD } \\
(\mathbf{n}=\mathbf{4 8 4})\end{array}$ & $\begin{array}{l}\text { p value Non-NAFLD } \\
\boldsymbol{v s . ~ N A F L D ~}\end{array}$ \\
\hline Age (years) & $53.0 \pm 11.9$ & $52.8 \pm 12.6$ & $53.3 \pm 10.5$ & 0.476 \\
\hline Male gender (yes) & $711(50.0)$ & $392(41.8)$ & $319(65.9)$ & $<0.001$ \\
\hline AST (U/L) & $22.8 \pm 8.6$ & $21.3 \pm 6.7$ & $25.8 \pm 10.8$ & $<0.001$ \\
\hline ALT (U/L) & $23.0 \pm 14.9$ & $18.2 \pm 8.4$ & $32.3 \pm 19.6$ & $<0.001$ \\
\hline Platelet $\left(\times 10^{9} / \mathrm{L}\right)$ & $237 \pm 65$ & $231 \pm 69$ & $249 \pm 56$ & $<0.001$ \\
\hline APRI & $0.27 \pm 0.13$ & $0.26 \pm 0.11$ & $0.29 \pm 0.15$ & $<0.001$ \\
\hline CRP $(\mathrm{mg} / \mathrm{dL})$ & $0.10(0.00-0.10)$ & $0.03(0.00-0.11)$ & $0.10(0.04-0.16)$ & 0.470 \\
\hline FLI $\geq 30$ (yes) & $432(30.4)$ & $114(12.2)$ & $318(65.7)$ & $<0.001$ \\
\hline FIB-4 index $\geq 2.67$ (yes) & $26(1.8)$ & $24(2.6)$ & $2(0.4)$ & 0.003 \\
\hline BMI (kg/mm $)$ & $22.7 \pm 3.5$ & $21.4 \pm 2.7$ & $25.3 \pm 3.4$ & $<0.001$ \\
\hline Hypertension (yes) & $252(17.7)$ & $128(13.7)$ & $124(25.6)$ & $<0.001$ \\
\hline Dyslipidemia (yes) & $196(13.8)$ & $107(11.4)$ & $89(18.4)$ & $<0.001$ \\
\hline Diabetes mellitus (yes) & $55(3.9)$ & $29(3.1)$ & $26(5.4)$ & 0.042 \\
\hline Exercise (yes) & $328(23.1)$ & $236(25.2)$ & $92(19.0)$ & 0.010 \\
\hline Smoking (yes) & $222(15.6)$ & $123(13.1)$ & $99(20.5)$ & $<0.001$ \\
\hline $\begin{array}{l}\text { Eating dinner within } 2 \text { hours } \\
\text { of going to bed (yes) }\end{array}$ & $305(21.5)$ & $175(18.7)$ & $130(26.9)$ & $<0.001$ \\
\hline
\end{tabular}

Table 1. Characteristics of subjects at baseline. Continuous variables except for CRP are expressed as means \pm standard deviations. CRP is expresssed as medians (interquartile ranges). They were compared by the unpaired $t$ test. Categorical variables are expressed as numbers (percentages), and were compared by the chi-squared test. ALT, alanine aminotransferase; APRI, aspartate aminotransferase to platelet ratio index; AST, aspartate aminotransferase; BMI, body mass index; CRP, C-reactive protein; FIB-4 index, fibrosis-4 index; FLI, fatty liver index; NAFLD, nonalcoholic fatty liver disease.

\begin{tabular}{|c|c|c|c|}
\hline & $\begin{array}{l}\text { At baseline } \\
(n=1,421)\end{array}$ & $\begin{array}{l}\text { At the last health } \\
\text { checkup }(n=1,421)\end{array}$ & p value \\
\hline Age (years) & $53.0 \pm 11.9$ & $57.6 \pm 12.4$ & $<0.001$ \\
\hline NAFLD (yes) & $484(34.1)$ & $461(32.4)$ & 0.148 \\
\hline $\operatorname{AST}(\mathrm{U} / \mathrm{L})$ & $22.8 \pm 8.6$ & $23.0 \pm 9.1$ & 0.425 \\
\hline $\operatorname{ALT}(\mathrm{U} / \mathrm{L})$ & $23.0 \pm 14.9$ & $22.3 \pm 14.1$ & 0.034 \\
\hline Platelet $\left(\times 10^{9} / \mathrm{L}\right)$ & $237 \pm 65$ & $231 \pm 59$ & $<0.001$ \\
\hline APRI & $0.27 \pm 0.13$ & $0.28 \pm 0.15$ & 0.004 \\
\hline $\mathrm{CRP}(\mathrm{mg} / \mathrm{dL})$ & $\begin{array}{l}0.10 \\
(0.00-0.10)\end{array}$ & $0.06(0.02-0.10)$ & 0.187 \\
\hline FLI $\geq 30$ (yes) & $432(30.4)$ & $437(30.8)$ & 0.772 \\
\hline FIB-4 index $\geq 2.67$ (yes) & $26(1.8)$ & $60(4.2)$ & $<0.001$ \\
\hline Body weight (kg) & $60.7 \pm 12.4$ & $60.8 \pm 12.4$ & 0.876 \\
\hline Exercise (yes) & $328(23.1)$ & $418(29.4)$ & $<0.001$ \\
\hline Smoking (yes) & $222(15.6)$ & $176(12.4)$ & $<0.001$ \\
\hline $\begin{array}{l}\text { Eating dinner within } 2 \\
\text { hours of going to bed (yes) }\end{array}$ & $305(21.5)$ & $288(20.3)$ & 0.284 \\
\hline
\end{tabular}

Table 2. Comparison of characteristics of subjects between baseline and the last health checkup. A total of 1,421 subjects (711 men and 710 women) was eligible for the present study. Continuous variables except for $\mathrm{CRP}$ are expressed as means \pm standard deviations. CRP is expresssed as medians (interquartile ranges). They were compared by the paired $t$ test. Categorical variables are expressed as numbers (percentages), and were compared by the McNemar test. ALT, alanine aminotransferase; APRI, aspartate aminotransferase to platelet ratio index; AST, aspartate aminotransferase; CRP, C-reactive protein; FIB-4 index, fibrosis-4 index; FLI, fatty liver index; NAFLD, nonalcoholic fatty liver disease.

In contrast, 127 of 484 (26.2\%, 5.7\%/year) subjects with NAFLD at baseline demonstrated NAFLD remission. Therefore, as shown in Table 2, the prevalence of NAFLD decreased from $34.1 \%$ at baseline to $32.4 \%$ at the last health checkup, although the difference was not statistically significant $(p=0.148)$. ALT levels decreased significantly $(\mathrm{p}=0.034)$. The frequency of exercise increased significantly, while that of smoking decreased significantly (both, $\mathrm{p}<0.001$ ).

Factors associated with NAFLD development. The changes in biochemical parameters and lifestyle habits were compared between subjects who developed NAFLD and those who remained non-NAFLD among subjects without NAFLD at baseline. As shown in Table 3, in subjects who developed NAFLD, ALT levels and the 


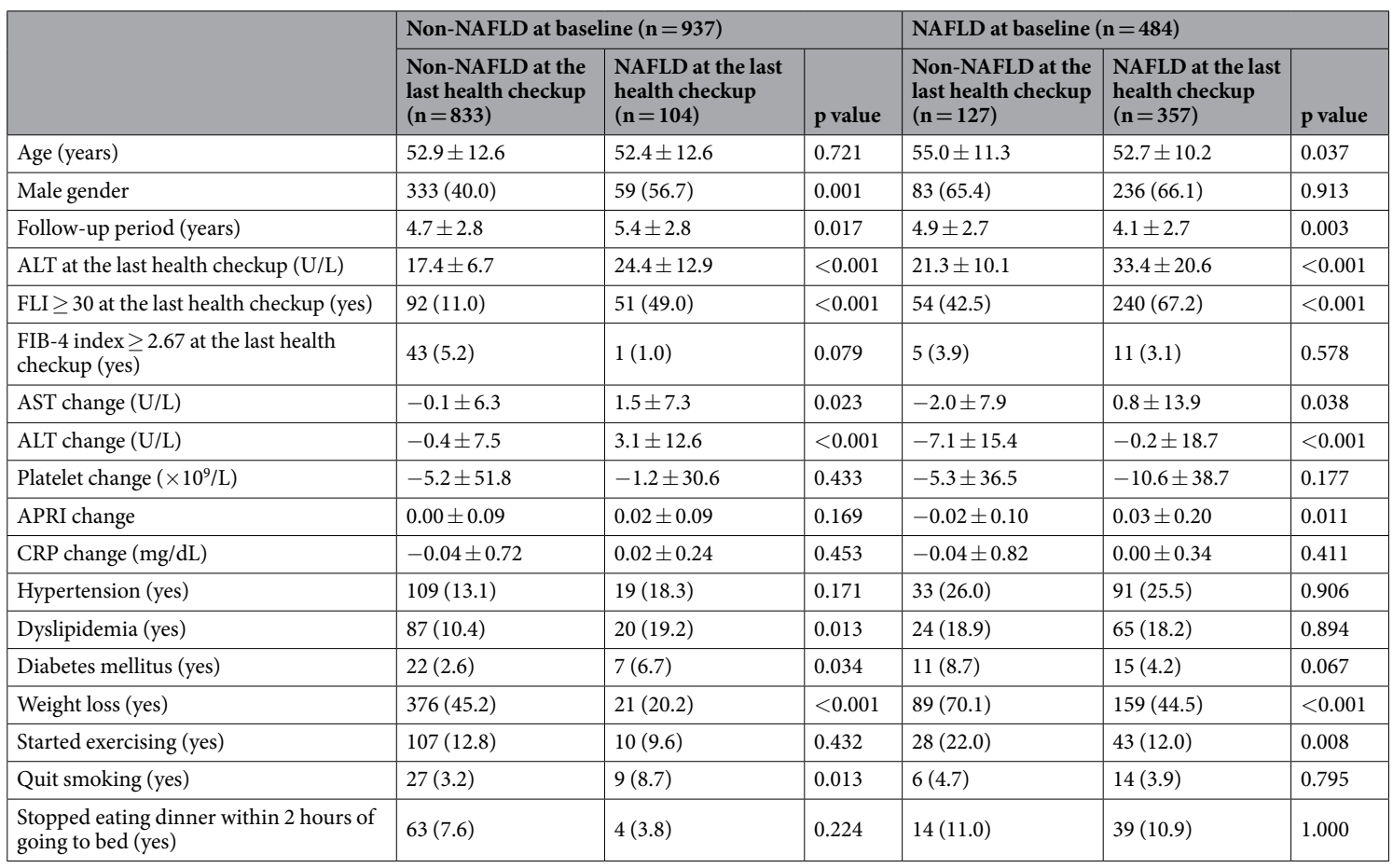

Table 3. Characteristics of subjects who developed NAFLD or demonstrated remission. Univariate analysis was performed. Continuous variables are expressed as means \pm standard deviations, and were compared by the unpaired t test. Categorical variables are expressed as numbers (percentages), and were compared by the chi-squared test. ALT, alanine aminotransferase; APRI, aspartate aminotransferase to platelet ratio index; AST, aspartate aminotransferase; CRP, C-reactive protein; FIB-4 index, fibrosis-4 index; FLI, fatty liver index; NAFLD, nonalcoholic fatty liver disease.

frequency of FLI $\geq 30$ at the last health checkup were significantly higher than those who remained non-NAFLD. Gender $(\mathrm{p}=0.001)$, follow-up period $(\mathrm{p}=0.017)$, AST change $(\mathrm{p}=0.023)$, ALT change $(\mathrm{p}<0.001)$, and the frequencies of dyslipidemia $(p=0.013)$, diabetes mellitus $(p=0.034)$, weight loss $(p<0.001)$, and quitting smoking $(\mathrm{p}=0.013)$ differed significantly between those who developed NAFLD and those who remained non-NAFLD. Among the changes of biochemical parameters, FLI change was most significantly associated with NAFLD development (Supplementary Table S1).

Multivariate analysis showed that male gender (adjusted odds ratio [AOR], 2.07; 95\% confidence interval [CI], 1.34-3.21), longer follow-up period (AOR, 1.11;95\% CI, 1.03-1.20), presence of dyslipidemia (AOR, 2.39; 95\% CI, 1.25-4.56), absence of weight loss (AOR, 0.30; 95\% CI, 0.18-0.50), and presence of quitting smoking (AOR, 2.86; 95\% CI, 1.24-6.62) were independently associated with the development of NAFLD (Table 4).

The association of weight change with NAFLD development was further analyzed. The larger weight gain was, the higher the frequency of NAFLD development was $(\mathrm{p}<0.001)$ (Fig. 2a). As shown in Fig. 3, the subjects who quit smoking during the follow-up period demonstrated large weight gain $(\geq 1 \%$ /year) significantly more frequently than the other subjects, including subjects who started smoking, continuing smoker, and non-smoker $(\mathrm{p}<0.001)$.

Factors associated with NAFLD remission. The changes in biochemical parameters and lifestyle habits were compared between subjects who demonstrated NAFLD remission and those remained NAFLD among subjects with NAFLD at baseline. As shown in Table 3, in subjects who demonstrated NAFLD remission, ALT levels and the frequency of FLI $\geq 30$ at the last health checkup were significantly lower than those remained NAFLD. Age $(p=0.037)$, follow-up period $(p=0.003)$, AST change $(p=0.038)$, ALT change $(p<0.001)$, APRI change $(\mathrm{p}=0.011)$, and the frequencies of weight loss $(\mathrm{p}<0.001)$ and starting to exercise $(\mathrm{p}=0.008)$ differed significantly between those who demonstrated NAFLD remission and those who remained NAFLD. Among the changes of biochemical parameters, FLI change $(p<0.001)$ and APRI change $(p=0.011)$ were independently associated with NAFLD remission (Supplementary Table S1).

Multivariate analysis showed that follow-up period (AOR, 1.12; 95\% CI, 1.03-1.21) and weight loss (AOR, $2.83 ; 95 \%$ CI, 1.80-4.44) were independently associated with NAFLD remission (Table 4 ). There was a mild but nonsignificant association between NAFLD remission and both older age (AOR, 1.02; 95\% CI, 1.00-1.04) and starting to exercise (AOR, 1.66; 95\% CI, 0.95-2.89). In sex-specific analysis, only in men, the independent association between NAFLD remission and starting to exercise was present (Table 5).

The association of weight change with NAFLD remission was further analyzed. The larger weight loss was, the higher the frequency of NAFLD remission was $(\mathrm{p}<0.001)$ (Fig. $2 b)$. 


\begin{tabular}{|l|l|l|l|l|l|l|}
\hline & \multicolumn{3}{|l|}{$\begin{array}{l}\text { Association of NAFLD } \\
\text { development (n= 937) }\end{array}$} & \multicolumn{3}{l|}{$\begin{array}{l}\text { Association of NAFLD remission } \\
(\mathbf{n}=\mathbf{4 8 4})\end{array}$} \\
\cline { 2 - 8 } & AOR & $\mathbf{9 5 \%}$ CI & p value & AOR & $\mathbf{9 5 \% ~ C I ~}$ & p value \\
\hline Age & 0.99 & $0.97-1.00$ & 0.130 & 1.02 & $1.00-1.04$ & 0.085 \\
\hline Male gender & 2.07 & $1.34-3.21$ & 0.001 & 0.97 & $0.61-1.53$ & 0.892 \\
\hline Follow-up period & 1.11 & $1.03-1.20$ & 0.006 & 1.12 & $1.03-1.21$ & 0.005 \\
\hline Hypertension & 1.27 & $0.66-2.47$ & 0.477 & 0.76 & $0.43-1.32$ & 0.331 \\
\hline Dyslipidemia & 2.39 & $1.25-4.56$ & 0.008 & 0.87 & $0.45-1.67$ & 0.671 \\
\hline Diabetes mellitus & 1.98 & $0.75-5.24$ & 0.169 & 1.88 & $0.75-4.72$ & 0.178 \\
\hline Weight loss & 0.30 & $0.18-0.50$ & $<0.001$ & 2.83 & $1.80-4.44$ & $<0.001$ \\
\hline Started exercising & 0.64 & $0.31-1.31$ & 0.222 & 1.66 & $0.95-2.89$ & 0.074 \\
\hline Quit smoking & 2.86 & $1.24-6.62$ & 0.014 & 1.04 & $0.37-2.95$ & 0.941 \\
\hline $\begin{array}{l}\text { Stopped eating dinner within } \\
\text { 2 hours of going to bed }\end{array}$ & 0.43 & $0.15-1.26$ & 0.124 & 1.20 & $0.60-2.37$ & 0.607 \\
\hline
\end{tabular}

Table 4. Factors associated with NAFLD development or remission. Multivariate analysis was performed using a logistic regression model, adjusted for all other factors in the table. AOR, adjusted odds ratio; NAFLD, nonalcoholic fatty liver disease.

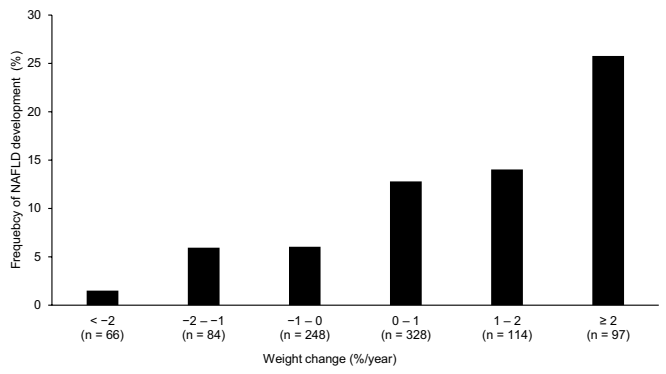

b

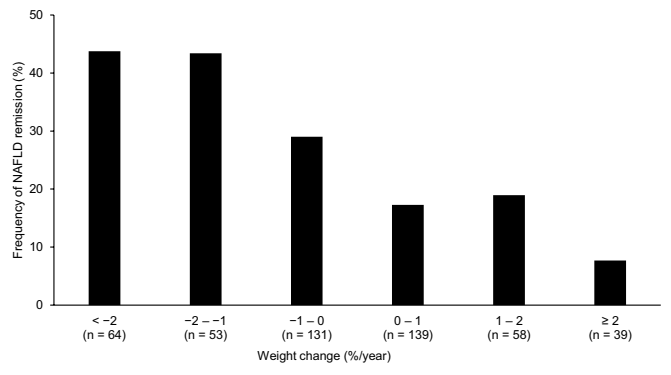

Figure 2. The association of weight change with NAFLD development and remission. (a) The larger weight gain was, the higher the frequency of NAFLD development was $(p<0.001)$. (b) The larger weight loss was, the higher the frequency of NAFLD remission was $(\mathrm{p}<0.001)$.

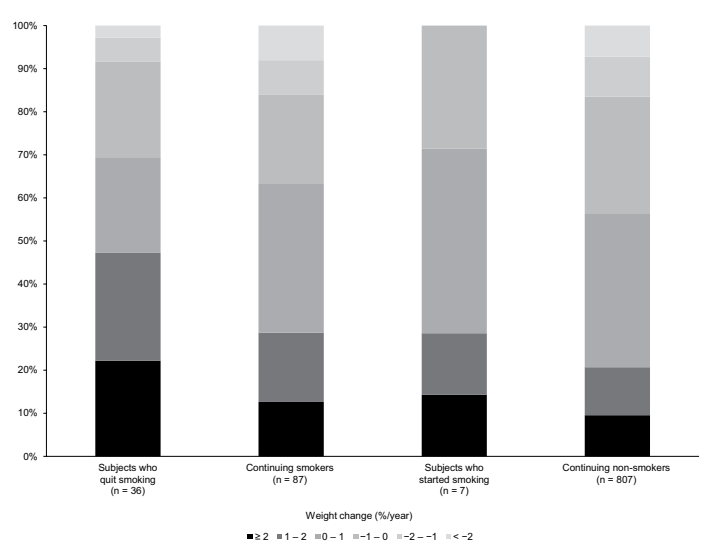

Figure 3. Weight change of subjects without nonalcoholic fatty liver disease at baseline, categorized according to changes of smoking habit. Subjects who quit smoking demonstrated large weight gain $(\geq 1 \%$ /year) significantly more frequently than the other subjects $(\mathrm{p}<0.001)$. 


\begin{tabular}{|c|c|c|c|c|c|c|c|c|c|c|c|c|}
\hline & \multicolumn{6}{|l|}{ men } & \multicolumn{6}{|c|}{ women } \\
\hline & \multicolumn{3}{|c|}{$\begin{array}{l}\text { Association of NAFLD } \\
\text { development }(n=392)\end{array}$} & \multicolumn{3}{|c|}{$\begin{array}{l}\text { Association of NAFLD } \\
\text { remission }(n=319)\end{array}$} & \multicolumn{3}{|c|}{$\begin{array}{l}\text { Association of NAFLD } \\
\text { development }(n=545)\end{array}$} & \multicolumn{3}{|c|}{$\begin{array}{l}\text { Association of NAFLD remission } \\
(\mathrm{n}=165)\end{array}$} \\
\hline & AOR & 95\% CI & p value & AOR & 95\% CI & p value & AOR & $95 \%$ CI & p value & AOR & $95 \% \mathrm{CI}$ & p value \\
\hline Age & 0.98 & $0.95-1.00$ & 0.070 & 1.03 & $1.00-1.06$ & 0.023 & 1.00 & $0.97-1.03$ & 0.982 & 0.99 & $0.95-1.03$ & 0.475 \\
\hline Follow-up period & 1.20 & $1.08-1.34$ & $<0.001$ & 1.13 & $1.03-1.25$ & 0.013 & 1.02 & $0.91-1.14$ & 0.767 & 1.07 & $0.93-1.24$ & 0.353 \\
\hline Hypertension & 1.30 & $0.51-3.32$ & 0.584 & 0.70 & $0.36-1.37$ & 0.300 & 1.40 & $0.53-3.72$ & 0.500 & 0.76 & $0.26-2.28$ & 0.629 \\
\hline Dyslipidemia & 2.64 & $1.00-6.97$ & 0.050 & 1.11 & $0.51-2.44$ & 0.788 & 1.71 & $0.65-4.49$ & 0.279 & 0.48 & $0.13-1.73$ & 0.263 \\
\hline Diabetes mellitus & 1.02 & $0.24-4.31$ & 0.978 & 1.78 & \begin{tabular}{|l|}
$0.60-5.29$ \\
\end{tabular} & 0.301 & 4.51 & $1.03-19.80$ & 0.046 & 1.36 & $0.20-9.28$ & 0.753 \\
\hline Weight loss & 0.13 & $0.05-0.32$ & $<0.001$ & 2.36 & $1.34-4.15$ & 0.003 & 0.54 & $0.27-1.06$ & 0.075 & 4.44 & $1.98-9.94$ & $<0.001$ \\
\hline Started exercising & 0.47 & $0.17-1.33$ & 0.154 & 2.38 & $1.25-4.53$ & 0.009 & 0.87 & $0.32-2.38$ & 0.788 & 0.41 & $0.12-1.49$ & 0.177 \\
\hline Quit smoking & 2.88 & $0.97-8.55$ & 0.056 & 1.22 & $0.41-3.64$ & 0.720 & 3.42 & $0.85-13.80$ & 0.083 & 0.00 & 0.00 -Infinity & 0.989 \\
\hline $\begin{array}{l}\text { Stopped eating dinner within } \\
2 \text { hours of going to bed }\end{array}$ & 0.27 & $0.06-1.23$ & 0.091 & 1.34 & $0.60-3.02$ & 0.480 & 0.84 & $0.19-3.71$ & 0.821 & 1.00 & $0.25-4.03$ & 0.997 \\
\hline
\end{tabular}

Table 5. Factors associated with NAFLD development or remission in sex-specific analysis. Multivariate analysis was performed using a logistic regression model, adjusted for all other factors in the table. AOR, adjusted odds ratio; NAFLD, nonalcoholic fatty liver disease.

\section{Discussion}

The present study showed that the prevalence of NAFLD at baseline was associated with lifestyle habits, such as exercise, smoking, and eating dinner within 2 hours of going to bed, in addition to BMI, gender, hypertension, dyslipidemia, and diabetes mellitus. All these lifestyle habits associations were reported previously ${ }^{14,15,20-23}$. Other lifestyle habits, including sleep duration and sitting time, have also been reported to be associated with NAFLD $^{24,25}$.

Many clinical trials have shown that patients with NAFLD benefit from weight $\operatorname{loss}^{3-6}$ and either dietary ${ }^{7-9}$ or exercise-related ${ }^{10-13}$ lifestyle interventions. There have been several longitudinal cohort studies on lifestyle habits involved in the development or remission of NAFLD. However, most of these studies assessed lifestyle habits at baseline but not changes in these habits during the study period ${ }^{14,16-18,26}$. We conducted a longitudinal cohort study to investigate the association of the development or remission of NAFLD with favorable changes in the lifestyle habits during follow-up period: weight loss, starting to exercise, quitting smoking, and stopping eating dinner within 2 hours of going to bed.

This study showed that change of body weight was significantly associated with both the development and remission of NAFLD. Quitting smoking was significantly associated with NAFLD development. In men, starting to exercise was significantly associated with NAFLD remission.

Several cohort studies have reported that change in body weight was significantly associated with both the development and remission of NAFLD ${ }^{27,28}$. The present study showed that weight change was correlated with both the frequency of NAFLD development and that of NAFLD remission. The frequency of NAFLD remission reached a plateau of $43 \%$ in subjects with $1-2 \%$ /year weight loss, and that of subjects with $\geq 2 \%$ /year weight loss was similarly $44 \%$. Musso et al. reported that $\geq 7 \%$ weight loss also improved histological disease activity in $\mathrm{NASH}^{4}$. However, the most effective rate of weight loss has not been well elucidated. Wong et al. conducted a clinical trial in which patients with NAFLD were provided with a 12-month lifestyle intervention, and then followed up for the next 5 years without intervention ${ }^{6}$. In patients with a baseline BMI of $\geq 25 \mathrm{~kg} / \mathrm{m}^{2}$, the mean change in body weight from baseline was $-7.0 \pm 4.8 \mathrm{~kg}$ at the end of the intervention, but only $-1.9 \pm 4.7 \mathrm{~kg}$ after 5 years. ALT levels temporarily dropped once but rebounded to the baseline level. This suggests that it is important to maintain the reduced body weight. Although the present study did not assess either the timing or speed of weight loss, it showed that approximately $40 \%$ of subjects who maintained $\geq 1 \%$ weight loss during the 4.6 -year follow-up period achieved NAFLD remission.

In this study, quitting smoking was independently associated with NAFLD development. This finding can be attributed to the significantly higher frequency of weight gain in subjects who quit smoking compared with the other subjects. Hamabe et al. reported that the odds ratio of NAFLD development among new quitters was 2.73 compared with nonsmokers ${ }^{14}$. Considerable weight gain after quitting smoking ${ }^{29}$ is probably due to increased appetite and reduced energy expenditure ${ }^{30}$. Hu et al. showed that the risk of type 2 diabetes was higher among recent quitters than among current smokers, and that the increase in the risk of type 2 diabetes was directly proportional to weight gain ${ }^{31}$. The same mechanism is probably applicable to NAFLD development after quitting smoking. Quitting smoking decreases both cardiovascular and all-cause mortality ${ }^{31}$. Thus, the recommendation of quitting smoking is important, while the guidance is necessary not to gain body weight and to avoid NAFLD development.

Starting to exercise was mildly but nonsignificantly associated with NAFLD remission in the analysis among all subjects of the present study. However, in men, starting to exercise was independently associated with NAFLD remission. Osaka et al. also reported in a 10-year cohort study that regular exercise at baseline, defined as participating in any kind of sports at least once a week, was associated with NAFLD remission only in men. Several clinical trials have shown the effectiveness of exercise in patients with NAFLD ${ }^{10,12}$. Further studies are needed to elucidate the difference between genders in terms of the effect of exercise on NAFLD. 
The present study showed that eating dinner within 2 hours of going to bed was significantly associated with the prevalence of NAFLD, while stopping of eating dinner within 2 hours of going to bed was not associated with either remission or development of NAFLD. Bo et al. showed that consuming a higher proportion of one's daily energy intake at dinner was associated with an increased risk of NAFLD, obesity, and metabolic syndrome ${ }^{26}$. This suggests that eating habits affect NAFLD development. More detailed data on eating habits are needed in order to investigate the association between eating habits and NAFLD.

The prevalence of NAFLD at baseline in the present study was $34.1 \%(484 / 1,421)$, which is higher than that of published studies in Japan $(9-30 \%)^{32,33}$. The subjects who were positive for HBsAg or anti-HCV Ab, or who drank $\geq 20 \mathrm{~g} /$ day of ethanol were excluded from the present study. When these subjects were included to the mother population, the prevalence of NAFLD is $24.7 \%(484 / 1,958)$. Among the subjects who had received health checkup only once during our study period and were excluded from the study, the prevalence of NAFLD was $30.9 \%(971 / 3,147)$. Thus, the prevalence of NAFLD of our study population was similar to that of general population or that of the population who were not eligible in the present study.

Our study population might have a relatively high health awareness, as indicated by the fact that the proportion of subjects with favorable lifestyle habits such as non-smoking and exercise significantly increased during the follow-up period, which allowed us to investigate the association of various lifestyle modifications with the development and remission of NAFLD. Moreover, we took advantage of the availability of the relatively large cohort and long follow-up period.

Our study had several limitations. First, since data on lifestyle habits were based on a self-reported questionnaire, it was uncertain when lifestyle habits were changed and how long these habits were continued. Second, the diagnosis of NAFLD was based on ultrasonography. Ultrasonography is a widely accepted imaging technique for the detection of fatty liver, because the both sensitivity and specificity of ultrasound for the detection of moderate-severe fatty liver compared to histology were high $(84.8 \% \text { and } 93.6 \% \text {, respectively })^{34}$. However, it may not detect mild steatosis and cannot differentiate NAFL from NASH. When diagnosing of fatty liver by ultrasonography, the four ultrasonographic findings (parenchymal brightness, liver-to-kidney contrast, deep beam attenuation, and vascular blurring) have been used. In the present study, we adopted the guideline for the ultrasonographic diagnosis of fatty liver published by the Japan Society of Ultrasonics in Medicine, in which fatty liver is diagnosed when one or more of the four findings ${ }^{35}$. However, the specificity of this protocol may be lower especially in obese subjects, because subjects with thick layers of subcutaneous fat can have deep beam attenuation, despite the absence of hepatic steatosis. The combination of the four findings can enable higher specificity. We used FLI, APRI, and fibrosis-4 (FIB-4) index to assess hepatic steatosis and fibrosis. FLI was significantly associated with the prevalence of NAFLD diagnosed by ultrasonography and its change was most significantly associated with the development and remission of NAFLD diagnosed by ultrasonography among the changes of biochemical parameters. This may indicate that the diagnostic method of NAFLD by ultrasonography is reliable. The value of APRI was low in most of the subjects. The subjects with FIB- 4 index $\geq 2.67$ are suspected to have advanced fibrosis and therefore $\mathrm{NASH}^{36}$. Among 484 subjects with NAFLD at baseline, the number of subjects with FIB- 4 index $\geq 2.67$ was only two $(0.4 \%)$ at baseline and $16(3.3 \%)$ at the last checkup. Among the 16 subjects, two (one remained NAFLD and one demonstrated NAFLD remission) had FIB-4 index $\geq 2.67$ at baseline and 14 (10 remained NAFLD and 4 demonstrated NAFLD remission) grew worse to have FIB- 4 index $\geq 2.67$ at the last checkup. Among 104 subjects with NAFLD development, none had FIB-4 index $\geq 2.67$ at baseline, and one grew worse to have FIB-4 index $\geq 2.67$ at the last checkup. These results suggested that the number of subjects with NASH was very small at baseline, but increased at the last checkup in the present study. It is needed to assess other fibrosis markers, liver stiffness or biopsy for the study of NASH in the future. Third, the ultrasonographic grade of hepatic steatosis was not considered in the present study. Mustapic et al. reported that patients with higher ultrasonographic grade of hepatic steatosis significantly more frequently had metabolic syndrome, while the grade of hepatic steatosis did not correlate with the presence of hepatic fibrosis ${ }^{37}$. However, there have been no reports on the clinical importance of the reduction in the number of ultrasonographic findings of NAFLD. It was difficult to define the boundary condition in the reduction in the number of ultrasonographic findings since the baseline numbers differed among the subjects. Thus, we set the boundary between none and one or more of the four characteristic ultrasonographic findings in NAFLD. Several cohort studies have also showed that disappearance of ultrasonographic findings for NAFLD was associated with a decreased risk of developing subclinical atherosclerosis and type 2 diabetes ${ }^{38,39}$. The clinical importance of the reduction in the number of ultrasonographic findings should be elucidated in the future studies.

In conclusion, the present longitudinal cohort study showed that weight change was correlated with both the frequency of NAFLD development and that of NAFLD remission. Quitting smoking induced NAFLD development, probably due to weight gain. Finally, starting to exercise was independently associated with NAFLD remission in men.

\section{Methods}

Study assessments. The health checkup included a standard questionnaire, physical examination, biochemical tests, and abdominal ultrasonography. The questionnaire assessed each patient's medical history of hypertension, dyslipidemia, and diabetes mellitus, as well as lifestyle habits such as alcohol consumption (how often and how much do you drink?), exercise (have you regularly exercised for over 30 minutes at a time, until you sweat lightly, at least twice weekly for over a year?), smoking (have you smoked during the past 6 months?), and eating dinner within 2 hours of going to bed (do you eat dinner within 2 hours before bedtime 3 or more times a week?). Height and weight were measured without shoes or heavy clothing, and BMI $\left(\mathrm{kg} / \mathrm{m}^{2}\right)$ was calculated. APRI and FIB-4 index were calculated by the following formula, respectively: APRI = (AST/upper limit of normal $\times 100) /$ platelet, FIB-4 index $=($ age $\times$ AST $) /\left(\right.$ platelet $\left.\times \mathrm{ALT}^{1 / 2}\right)$. The upper limit of normal AST levels was $38 \mathrm{U} / \mathrm{L}$. FLI was calculated using BMI (in $\mathrm{kg} / \mathrm{m}^{2}$ ), waist circumference (WC, in $\mathrm{cm}$ ), triglycerides (TG, in $\mathrm{mg} /$ 
$\mathrm{dL})$ and gamma-glutamyl transferase (GGT, in $\mathrm{U} / \mathrm{L}): \mathrm{FLI}=\left[\mathrm{e}^{(0.953 \times \ln (\mathrm{TG})+0.139 \times \mathrm{BMI}+0.718 \times \ln (\mathrm{GGT})+0.053 \times \mathrm{WC}-15.745)}\right] /$ $\left[1+\mathrm{e}^{(0.953 \times \ln (\mathrm{TG})+0.139 \times \mathrm{BMI}+0.718 \times \ln (\mathrm{GGT})+0.053 \times \mathrm{WC}-15.745)}\right] \times 100$, where $\ln$ is the natural logarithm and e Euler's number.

NAFLD was defined as fatty liver without excessive alcohol consumption (ethanol consumption $\geq 20 \mathrm{~g} /$ day) or other chronic liver disease such as hepatitis B or C. In accordance with guideline published by the Japan Society of Ultrasonics in Medicine ${ }^{35}$, the diagnosis of fatty liver was required one or more of the following four ultrasonographic findings: parenchymal brightness, liver-to-kidney contrast, deep beam attenuation, and vascular blurring. Medical technologists performed all ultrasonography procedures using the Aplio SSA-700A (Toshiba, Tokyo, Japan) or Xario 200 Platinum (Canon, Tokyo, Japan). Two gastroenterologists who were blinded to the subjects' clinical data reviewed all the ultrasonographic images and made the diagnosis of fatty liver (positive for one or more of the four characteristic ultrasonographic findings) or non-fatty liver (positive for none of the four findings). When two opinions varied, the diagnosis was decided by their discussion (kappa value for the intra-observer reliability was $0.980 ; 95 \% \mathrm{CI}, 0.972-0.988$ ). NAFLD development was defined as the transition from non-NAFLD (positive for none of the four characteristic ultrasonographic findings) to NAFLD (positive for one or more of the four findings) during the follow-up period which was defined as the period between the first and last health checkups. NAFLD remission was defined as the transition from NAFLD (positive for one or more of the four findings) to non-NAFLD (positive for none of the four findings) during the period.

We examined the factors associated with development or remission of NAFLD by comparing each patient's data at the first health checkup (at baseline) with those at the last health checkup. With respect to changes in lifestyle habits at the last health checkup from baseline, "started exercising", "quit smoking", "stopped eating dinner within 2 hours of going to bed", and "lost weight" were defined based on changes during the follow-up period. The average of weight change (\%/year) was calculated as follows: (weight change during the follow-up period/ body weight at baseline)/the follow-up period. Additionally, sex-specific analysis was performed on the factors associated with development or remission of NAFLD.

The study protocol including the method of opt-out consent was approved by the Institutional Review Board at Nagoya University Graduate School of Medicine (2019-0024) and Japanese Red Cross Nagoya Daiichi Hospital (2018-238), and the study was conducted in accordance with the concepts outlined in the Declaration of Helsinki. Informed consent was obtained in the form of opt-out on the website of Japanese Red Cross Nagoya Daiichi Hospital.

Health guidance. The results of health checkup were given to all subjects on the checkup day at Japanese Red Cross Nagoya Daiichi Hospital. In addition, health guidance was provided to all subjects unless they denied it, by healthcare staff, consisting of physician, public health nurse, or nutritionist. Independent advice was given using leaflets. When received the final results by mail within 2 weeks after health checkup, specific health guidance was recommended for subjects in high risk with metabolic syndrome. Subjects who requested were provided additional continuous support for 3 months or longer in response to individual risks via interview or telephone.

Statistical analysis. Continuous variables except for CRP were expressed as means \pm standard deviations. $\mathrm{CRP}$ was expressed as medians and interquartile ranges. They were analyzed using the unpaired and paired $t$ tests. Categorical variables were expressed as numbers and percentages, and were analyzed using the chi-squared test and the McNemar test. The Cochran-Armitage test was used to assess the trend in the frequencies of development or remission of NAFLD based on weight change. Multivariate analysis was performed using a logistic regression model. After adjusting for age, gender, follow-up period, hypertension, dyslipidemia, diabetes mellitus, starting exercise, quitting smoking, stopping eating dinner within 2 hours of going to bed, and weight loss, the AOR and 95\% CI were calculated. The data were analyzed using EZR (Saitama Medical Center, Jichi Medical University, Saitama, Japan), which is a graphical user interface for R (The R Foundation for Statistical Computing, Vienna, Austria, version 3.5.3). More precisely, it is a modified version of the $\mathrm{R}$ commander (version 2.5-1) designed to add statistical functions frequently used in biostatistics ${ }^{40}$. All the reported p values were two-tailed, and a value of $\mathrm{p}<0.05$ was considered statistically significant.

Received: 12 July 2019; Accepted: 18 December 2019;

Published online: 16 January 2020

\section{References}

1. Younossi, Z. et al. Global burden of NAFLD and NASH: trends, predictions, risk factors and prevention. Nature reviews. Gastroenterology \& hepatology 15, 11-20 (2018).

2. Chalasani, N. et al. The diagnosis and management of non-alcoholic fatty liver disease: practice Guideline by the American Association for the Study of Liver Diseases, American College of Gastroenterology, and the American Gastroenterological Association. Hepatology 55, 2005-2023 (2012).

3. Promrat, K. et al. Randomized controlled trial testing the effects of weight loss on nonalcoholic steatohepatitis. Hepatology 51, 121-129 (2010).

4. Musso, G., Cassader, M., Rosina, F. \& Gambino, R. Impact of current treatments on liver disease, glucose metabolism and cardiovascular risk in non-alcoholic fatty liver disease (NAFLD): a systematic review and meta-analysis of randomised trials. Diabetologia 55, 885-904 (2012).

5. Vilar-Gomez, E. et al. Weight Loss Through Lifestyle Modification Significantly Reduces Features of Nonalcoholic Steatohepatitis. Gastroenterology 149, 367-378.e365; quiz e314-365 (2015).

6. Wong, V. W. et al. Beneficial effects of lifestyle intervention in non-obese patients with non-alcoholic fatty liver disease. Journal of hepatology 69, 1349-1356 (2018).

7. Bozzetto, L. et al. Liver fat is reduced by an isoenergetic MUFA diet in a controlled randomized study in type 2 diabetic patients. Diabetes care 35, 1429-1435 (2012).

8. Ryan, M. C. et al. The Mediterranean diet improves hepatic steatosis and insulin sensitivity in individuals with non-alcoholic fatty liver disease. Journal of hepatology 59, 138-143 (2013). 
9. Trovato, F. M., Catalano, D., Martines, G. F., Pace, P. \& Trovato, G. M. Mediterranean diet and non-alcoholic fatty liver disease: the need of extended and comprehensive interventions. Clinical nutrition 34, 86-88 (2015).

10. Keating, S. E., Hackett, D. A., George, J. \& Johnson, N. A. Exercise and non-alcoholic fatty liver disease: a systematic review and meta-analysis. Journal of hepatology 57, 157-166 (2012).

11. Sullivan, S., Kirk, E. P., Mittendorfer, B., Patterson, B. W. \& Klein, S. Randomized trial of exercise effect on intrahepatic triglyceride content and lipid kinetics in nonalcoholic fatty liver disease. Hepatology 55, 1738-1745 (2012)

12. Berzigotti, A., Saran, U. \& Dufour, J. F. Physical activity and liver diseases. Hepatology 63, 1026-1040 (2016).

13. Zhang, H. J. et al. Effects of Moderate and Vigorous Exercise on Nonalcoholic Fatty Liver Disease: A Randomized Clinical Trial. JAMA internal medicine 176, 1074-1082 (2016).

14. Hamabe, A. et al. Impact of cigarette smoking on onset of nonalcoholic fatty liver disease over a 10-year period. Journal of gastroenterology 46, 769-778 (2011).

15. Nishi, T., Babazono, A., Maeda, T., Imatoh, T. \& Une, H. Effects of Eating Fast and Eating Before Bedtime on the Development of Nonalcoholic Fatty Liver Disease. Population health management 19, 279-283 (2016).

16. Suzuki, A. et al. Effect of changes on body weight and lifestyle in nonalcoholic fatty liver disease. Journal of hepatology 43, 1060-1066 (2005).

17. Okamoto, M. et al. Cigarette smoking is a risk factor for the onset of fatty liver disease in nondrinkers: A longitudinal cohort study. PloS one 13, e0195147 (2018).

18. Osaka, T. et al. Nonalcoholic fatty liver disease remission in men through regular exercise. Journal of clinical biochemistry and nutrition 62, 242-246 (2018).

19. Nakao, Y. M. et al. Effectiveness of nationwide screening and lifestyle intervention for abdominal obesity and cardiometabolic risks in Japan: The metabolic syndrome and comprehensive lifestyle intervention study on nationwide database in Japan (MetS ACTION-J study). PloS one 13, e0190862 (2018).

20. Wong, V. W. et al. Prevalence of non-alcoholic fatty liver disease and advanced fibrosis in Hong Kong Chinese: a population study using proton-magnetic resonance spectroscopy and transient elastography. Gut 61, 409-415 (2012).

21. Fung, J. et al. High prevalence of non-alcoholic fatty liver disease in the Chinese - results from the Hong Kong liver health census. Liver international 35, 542-549 (2015).

22. Younossi, Z. M. et al. Global epidemiology of nonalcoholic fatty liver disease-Meta-analytic assessment of prevalence, incidence, and outcomes. Hepatology 64, 73-84 (2016).

23. Bae, J. C. et al. Regular exercise is associated with a reduction in the risk of NAFLD and decreased liver enzymes in individuals with NAFLD independent of obesity in Korean adults. PloS one 7, e46819 (2012).

24. Miyake, T. et al. Short sleep duration reduces the risk of nonalcoholic fatty liver disease onset in men: a community-based longitudinal cohort study. Journal of gastroenterology 50, 583-589 (2015).

25. Ryu, S. et al. Relationship of sitting time and physical activity with non-alcoholic fatty liver disease. Journal of hepatology 63 , 1229-1237 (2015).

26. Bo, S. et al. Consuming more of daily caloric intake at dinner predisposes to obesity. A 6-year population-based prospective cohort study. PloS one 9 , e108467 (2014).

27. Hamaguchi, M. et al. The metabolic syndrome as a predictor of nonalcoholic fatty liver disease. Annals of internal medicine 143, $722-728$ (2005).

28. Zelber-Sagi, S. et al. Predictors for incidence and remission of NAFLD in the general population during a seven-year prospective follow-up. Journal of hepatology 56, 1145-1151 (2012).

29. Aubin, H. J., Farley, A., Lycett, D., Lahmek, P. \& Aveyard, P. Weight gain in smokers after quitting cigarettes: meta-analysis. BMJ 345, e4439 (2012)

30. Harris, K. K., Zopey, M. \& Friedman, T. C. Metabolic effects of smoking cessation. Nature reviews. Endocrinology 12, 299-308 (2016).

31. Hu, Y. et al. Smoking Cessation, Weight Change, Type 2 Diabetes, and Mortality. The New England journal of medicine 379, 623-632 (2018).

32. Jimba, S. et al. Prevalence of non-alcoholic fatty liver disease and its association with impaired glucose metabolism in Japanese adults. Diabetic medicine 22, 1141-1145 (2005).

33. Hashimoto, E. \& Tokushige, K. Prevalence, gender, ethnic variations, and prognosis of NASH. Journal of gastroenterology 46(Suppl 1), 63-69 (2011).

34. Hernaez, R. et al. Diagnostic accuracy and reliability of ultrasonography for the detection of fatty liver: a meta-analysis. Hepatology 54, 1082-1090 (2011).

35. Guideline for Abdominal Ultrasound in Cancer Screening and Health Checkups in Japan. the Japan Society of Ultrasonics in Medicine https://www.jsum.or.jp/committee/diagnostic/pdf/fukubu_42-2.pdf (2015).

36. Shah, A. G. et al. Comparison of noninvasive markers of fibrosis in patients with nonalcoholic fatty liver disease. Clinical gastroenterology and hepatology 7, 1104-1112 (2009).

37. Mustapic, S. et al. Ultrasound Grade of Liver Steatosis Is Independently Associated with the Risk of Metabolic Syndrome. Canadian journal of gastroenterology \& hepatology 2018, 8490242 (2018).

38. Sinn, D. H. et al. Persistent Nonalcoholic Fatty Liver Disease Increases Risk for Carotid Atherosclerosis. Gastroenterology 151, 481-488 (2016)

39. Yamazaki, H., Tsuboya, T., Tsuji, K., Dohke, M. \& Maguchi, H. Independent Association Between Improvement of Nonalcoholic Fatty Liver Disease and Reduced Incidence of Type 2 Diabetes. Diabetes care 38, 1673-1679 (2015).

40. Kanda, Y. Investigation of the freely available easy-to-use software 'EZR' for medical statistics. Bone marrow transplantation 48 , $452-458(2013)$

\section{Acknowledgements}

The authors thank Chiemi Shibata and Michiko Fujimoto of Health control Center at Japanese Red Cross Nagoya Daiichi Hospital for their assistance with data collection.

\section{Author contributions}

Study design: N. Yoshioka. Analysis and interpretation of data: N. Yoshioka. Drafting of manuscript: N. Yoshioka. Critical revision: M. Ishigami, T. Ito, Y. Ishizu, T. Kuzuya, T. Honda, T. Ishikawa, and M. Fujishiro. Collection of data: N. Yoshioka, H. Sumi, M. Doisaki, T. Yamaguchi, Y. Watanabe, and J. Haruta.

\section{Competing interests}

The authors declare no competing interests. 


\section{Additional information}

Supplementary information is available for this paper at https://doi.org/10.1038/s41598-019-57369-9.

Correspondence and requests for materials should be addressed to M.I.

Reprints and permissions information is available at www.nature.com/reprints.

Publisher's note Springer Nature remains neutral with regard to jurisdictional claims in published maps and institutional affiliations.

(c) (i) Open Access This article is licensed under a Creative Commons Attribution 4.0 International License, which permits use, sharing, adaptation, distribution and reproduction in any medium or format, as long as you give appropriate credit to the original author(s) and the source, provide a link to the Creative Commons license, and indicate if changes were made. The images or other third party material in this article are included in the article's Creative Commons license, unless indicated otherwise in a credit line to the material. If material is not included in the article's Creative Commons license and your intended use is not permitted by statutory regulation or exceeds the permitted use, you will need to obtain permission directly from the copyright holder. To view a copy of this license, visit http://creativecommons.org/licenses/by/4.0/.

(c) The Author(s) 2020 